\title{
Vino novedoso, ¿a quién dirigirlo?
}

\author{
HAROLD MARTÍN-CARO MALAVÉ* \\ HELLEN MÉNDEZ MARTÍNEZ** \\ CRISTINA OLARTE-PASCUAL ${ }^{* * *}$
}

pp. $74-82$

* Doctor en Estadística. Universidad de La Rioja, Logroño, España. E-mail: hamartin@unirioja.es. ORCID: 00oo-0003-1310-4522. Google Scholar: https://scholar.google.es/citations?user=BnozhU8AAAAJ\&hl=es.

**Máster en Viticultura, Enología y Dirección de Empresas Vitivinícolas. Universidad de La Rioja, Logroño, España. E-mail: hemendez@unirioja.es ORCID: 0000-0001-8680-7144. Google Scholar: https://scholar.google.es/citations?user=WoBeol4AAAAJ\&h=es.

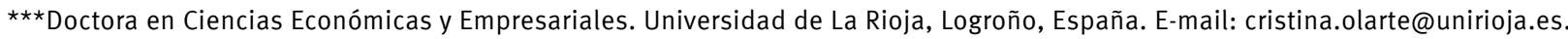
ORCID: 0000-0002-9853-0266. Google Scholar: https://scholar.google.es/citations?hl=es\&user=sLRzywYAAAAJ. 


\section{COMO CITAR ESTE ARTÍCULO \\ How to cite this article:}

Martín-Caro, H., Méndez, H. y Olarte-Pascual, C. (2020). Vino novedoso, ¿a quién dirigirlo? Revista Perspectiva Empresarial, 7(2), 74-82.

Recibido: 19 de agosto de 2020

Aceptado: 06 de noviembre de 2020
RESUMEN La innovación y la segmentación del mercado son factores de éxito ante mercados maduros, competitivos y controlados como lo es el del vino. En este trabajo se aborda la segmentación del mercado del vino ante un nuevo vino tinto espumoso natural. Se utilizan variables que determinan la intención de compra tales como actitudes y consumo de vino, beneficios y emociones causadas por el vino, edad, género, nivel educativo e ingresos. Como resultado se obtuvieron cuatro segmentos diferenciados: uno más abierto a innovaciones como el 'optimista'; el 'dispuesto'; el “exigente moderado" que exige con cierta racionalidad y el 'pretencioso' que es el más exigente, pero que no es un gran consumidor. Se concluye que las innovaciones deben dirigirse hacia los segmentos denominados 'optimista' y 'dispuesto'.

PALABRAS CLAVE análisis de componentes principales, análisis de cluster, escenario, segmento, conglomerado.

\section{Novelty Wine, who to target?}

ABSTRACT Innovation and market segmentation are success factors in mature, competitive and controlled markets such as the wine market. This paper addresses the segmentation of the wine market for a new natural sparkling red wine. Variables that determine purchase intention such as attitudes and wine consumption, benefits and emotions caused by wine, age, gender, educational level and income are used. As a result, four differentiated segments were obtained: the 'optimistic', more open to innovations; the 'willing'; the "moderate demanding" who demands with certain rationality and the "pretentious' who is the most demanding, but who is not a big consumer. It is concluded that innovations should be directed towards the segments called 'optimistic' and 'willing'.

KEYWORDS Main component analysis, cluster analysis, scenario, segment, cluster. 


\section{Vinho innovador, a quem encaminhá-lo?}

RESUMO Inovação e segmentação de mercado são fatores de sucesso em mercados maduros, competitivos e controlados como o vinho. Este trabalho aborda a segmentação do mercado vitivinícola face a um novo espumante tinto natural. São utilizadas variáveis que determinam a intenção de compra, como atitudes e consumo de vinho, benefícios e emoções decorrentes do vinho, idade, sexo, escolaridade e renda. Como resultado, foram obtidos quatro segmentos diferenciados: um mais aberto a inovações como 0 ‘otimista'; o ‘querer'; o “exigente moderado" que exige com certa racionalidade e o “pretensioso" quem é o mais exigente, mas não é um grande consumidor. Conclui-se que as inovações devem ser direcionadas aos segmentos ditos 'otimistas' e ‘dispostos'.

PALAVRAS-CHAVE análise de componentes principais, análise de cluster, cenário, segmento, conglomerado. 


\section{Introducción}

La globalización e internacionalización, las grandes producciones de grandes empresas, consumidores exigentes con gran cantidad de información disponible y conscientes de lo que buscan, hacen que el sector alimentario sea altamente competitivo (Barrena, García y López-Mosquera, 2016). Por ello la innovación es una opción importante, aunque no sencilla porque pese a los valiosos esfuerzos académicos y empresariales para el desarrollo de nuevos productos la tasa de rechazo es cercana al $70 \%$ (Moreno y Munuera, 2012). Muchas empresas gastan miles de millones de dólares fabricando mousetrap (productos engañosos), rechazados luego por los consumidores entre un 40 y $90 \%$ según su categoría (Gourville, 2006).

El sector del vino es aún más complicado, pues es altamente competitivo; tan solo en España existen 4373 bodegas (OEMV, 2018), siendo más de 80000 bodegas en el mundo las que producen 250 millones de litros por año (OIV, 2018); es maduro debido a su antigüedad y evolución; con avances en los últimos años por la alta competencia y diversidad de estilos; es sumamente controlado, sobre todo en Europa donde existen regulaciones impuestas por gobiernos y organizaciones supranacionales (Juaneda-Ayensa et al., 2019).

Por otra parte el consumo mundial de vino mostró un crecimiento interanual de $0,79 \%$ hacia 2017, aunque decreció a una tasa de-0,28\% desde ese mismo año. En España el descenso fue más marcado: $-1,79 \%$ entre 1995 y 2007 , de 15336 a 13100 miles de hectolitros; -1,30 \% entre 2007 y 2017, de 13100 a 10300 miles de hectolitros (OIV, 2018).

Las causas de este decrecimiento para los consumidores pueden ser muchas, destacando entre ellas: no les gusta el sabor ni el grado de alcohol; es una bebida para tomar en las comidas y en ocasiones especiales; es difícil e incómodo de beber; no refresca y no se sienten atraídos por su consumo (prefiriendo otras bebidas como, por ejemplo, refrescos y cerveza).

Un nuevo vino, con características diferentes, pudiera captar nuevos consumidores. Por esta razón, se plantea la elaboración de un nuevo vino espumoso tinto natural. Una innovación puede generar cambios en el mercado debido a que los consumidorestradicionales podrían rechazarla, pero contrariamente los más abiertos la podrían consumir. Así, es importante determinar los grupos propensos a aceptarla y dirigir a ellos dicha innovación.

El análisis de este trabajo se basa en una investigación realizada en Logroño, en una muestra de 500 individuos en la que se midió la opinión de consumidores sobre el nuevo vino tinto espumoso tinto natural (Olarte, Pelegrín and Reinares, 2017), aplicando una cata y un cuestionario que surgió de una investigación previa con 44 expertos; basada en cata del producto y su respectivo análisis sensorial, definiendo los beneficios y razones de compra para beber el nuevo vino (Juaneda-Ayensa et al., 2019; Olarte, Pelegrín and Reinares, 2017).

El vino es elaborado con la variedad tempranillo y con el método tradicional, el Cava, su grado alcohólico es de 12,5을 (similar al de los Cava y a otros vinos espumosos).

A la base de datos del estudio se aplicaron métodos estadísticos de análisis de datos para conformar e identificar segmentos de mercado que se diferencien en su actitud ante este nuevo producto; con el fin de que puedan ser utilizados en posibles estrategias de producción y marketing.

Desde la publicación de Wendell R. Smith, en 1956, "Product Differentiation and Market Segmentation as Alternative Marketing Strategies", prácticamente no existe un análisis de mercado sin su respectiva segmentación y su utilidad; una vez clasificados los individuos en los segmentos de interés hay que, entre otras estrategias, cuantificarlos y entenderlos para fijar estrategias de comunicación y marketing idóneas, optimizar la distribución y ubicación del producto, así como fijar el precio adecuado con el objetivo final de maximizar las ventas y/o ganancias.

\section{Metodología}

Con el objetivo de saber si todos los consumidores se comportan igual ante una innovación o si constituyen distintos grupos, se 
diseñó una investigación sobre una muestra de 500 individuos a los que se les dio a degustar el producto y se les pasó un cuestionario con el fin de segmentar la muestra e identificar tanto actitudes ante el producto como otras variables de tipo socioeconómicas y demográficas.

Como ya se mencionó, el producto consiste en un vino tinto espumoso natural. Cabe destacar que a nivel mundial hay pocos vinos tintos espumosos, siendo el Lambrusco uno de los más conocidos; elaborado en Italia en varias denominaciones e indicaciones de origen en la región de EmiliaRomaña y Lombardía. Otro de ellos es el Brachetto d'Acqui en el Piamonte italiano, también en Australia, a base de shiraz. A diferencia de estos, el vino en cuestión es elaborado con el método tradicional como el Cava y el Champagne.

El vino en estudio cumple con algunas características -sugeridas por diversos entrevistados en un estudio de la OEMV (2014)para incrementar el consumo tales como bajo contenido alcohólico, menos tánico y fácil de abrir; aspectos similares al Lambrusco, que particularmente es más consumido por mujeres y jóvenes, aunque este tipo de vino no es tomado muy en serio por los conocedores.

El cuestionario consta de 64 variables (de tipo cualitativa ordinal), relativas a opinión y actitud sobre el vino, medidas en una escala de Likert de 0 a 10, agrupadas en cuatro categorías: intención y motivación de consumo, y norma social (16); emociones que produce el vino en el consumidor (20); beneficios que genera el vino (24) e importancia en la compra de vino (4); al igual que varias demográficas (edad, género, ocupación, nivel de estudios finalizados e ingreso mensual), además de consumo semanal de vino. Primero se realizó un análisis de consistencia de las variables para garantizar que todos los ítems midan un mismo constructo y estén correlacionados entre sí (FríasNavarro, 2014), aplicando el alfa de Cronbach. Luego se aplicó un análisis de componentes principales - ACP - para reducir la cantidad de variables, manteniendo un alto porcentaje de la variabilidad global de los datos con la ventaja de que estas nuevas "variables latentes" fueron incorrelacionadas con lo que se evitó la posible redundancia en los datos. Posteriormente se aplicó un análisis cluster - ACpara clasificar los individuos en grupos homogéneos en su interior y heterogéneos entre ellos. En este sentido se generaron varios escenarios y se determinó el idóneo para el estudio. En resumen, el procedimiento utilizado se indica a continuación:

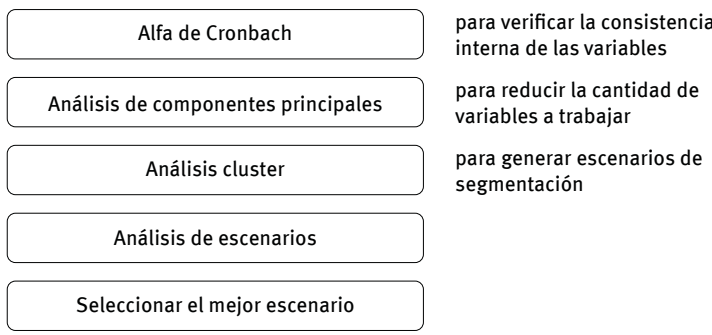

Figura 1. Procedimiento estadístico. Fuente: elaboración propia por parte de los autores.

Como la aceptación de una innovación está determinada por variables tales como beneficios sensoriales y de precios, norma social y factores afectivos, así como emociones tanto positivas como negativas (Olarte, Pelegrín and Reinares, 2017; García-Milon etal., 2019), las variables de opinión y actitud sobre el vino se agruparon en: intención y motivación de consumo; norma social; emociones y beneficios. Con respecto al resto de variables, 'sexo', "nivel de estudios finalizados", "ocupación principal actual" e "ingreso mensual" estas se mantuvieron; mientras que "edad" y "consumo semanal de vino" se agruparon en clases categóricas.

\section{Resultados}

En primer lugar se realizó un análisis de consistencia de las variables, para ello se agruparon las variables en 4 dimensiones: dimensión 1 (intención, motivación y norma social); dimensión $2 \mathrm{a}$ (emociones positivas); dimensión $2 \mathrm{~b}$ (emociones negativas) y dimensión 3 (beneficios).

Para determinar las emociones positivas y negativas se adaptó la escala PANAS (Watson, Clark and Tellegen, 1988). Se calculó el alfa de Cronbach estandarizado, encontrándose en todos los casos valores por encima de 0,87 ; siendo altos para varios autores como Gliem y Gliem (2003) y George y Mallery (2003), por lo que se trabajó con todas las variables. 
Para simplificar la construcción de los segmentos se agruparon las 64 variables, generando 13 nuevas "variables compuestas": 5 de la dimensión 1 (que incluye intención de compra, norma social, facilidad de uso, motivación hedónica, percepción de calidad); 1 de la dimensión 2a; 1 de la dimensión 2b; 5 de la dimensión 3 (que incluye beneficios sensoriales, beneficios a la salud, beneficios sociales, beneficios para la región y otros beneficios) y 1 sobre importancia en compra de vino. En el ACP se utilizaron dichas variables, así como "consumo semanal de vino". Se excluyeron las variables sociodemográficas. De este análisis se tomaron las primeras 9 como componentes, que recogen el 90,169 \% de la variabilidad total respectivamente; con base en ello se realizó el $\mathrm{AC}$, con lo cual se trabajó con menos variables; además, por la estructura de estos componentes, se detectaron patrones ocultos difíciles de observar en las variables originales tal como lo aplican Granato et al. (2018) y Khodasevich et al. (2017).

A las 9 variables componentes y las variables sociodemográficas se les aplicó el AC en el que se generaron varios escenarios, alternando el tratamiento de las mismas; tomándolas de forma original, estandarizadas u homogeneizadas; en cada escenario se contemplaron subescenarios de 2, 3, 4 y 5 conglomerados o segmentos. El escenario que mejor discrimina o diferencia utiliza las componentes de forma original y las variables sociodemográficas homogeneizadas.

En el AC el método aplicado fue el de Ward, que es un método jerárquico para no restringir la solución a un número prefijado de segmentos. Este método ha tenido mejor desempeño que otros (Ferreira and Hitchcock, 2009; Hands and Everitt, 1987; Kuiper and Fisher, 1975; Blashfield, 1976).

A continuación se muestran las figuras de este escenario, en las telas de araña se observan los valores promedios de cada variable generada de cada segmento para los subescenarios de 5 , 4, 3 y 2 segmentos; mostrando la evolución de la conformación de los mismos; luego, el tamaño de cada segmento. Se identifican con colores la evolución de la conformación de los mismos.
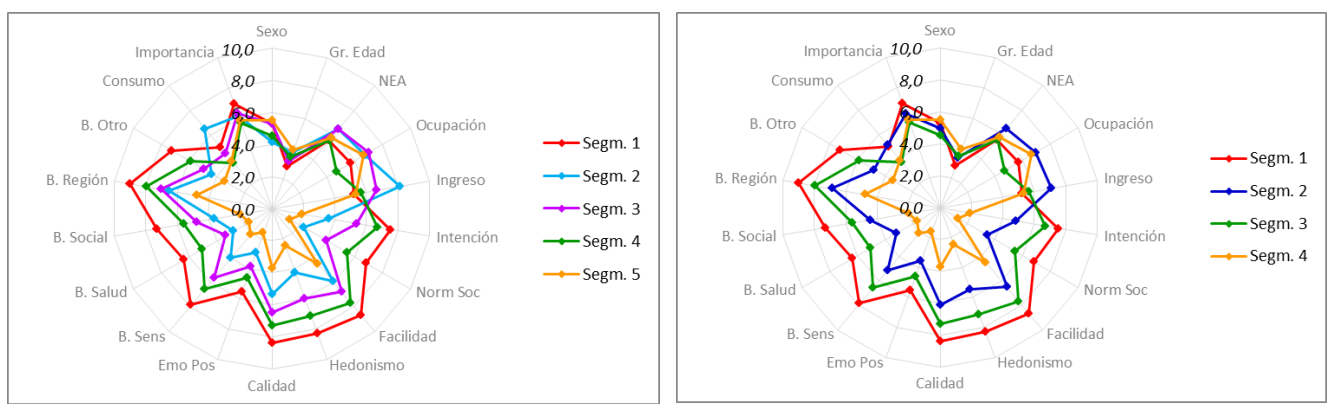

Figuras 2-3. Comportamiento de variables según segmento ( 5 y 4 segmentos respectivamente). Fuente: elaboración propia por parte de los autores.
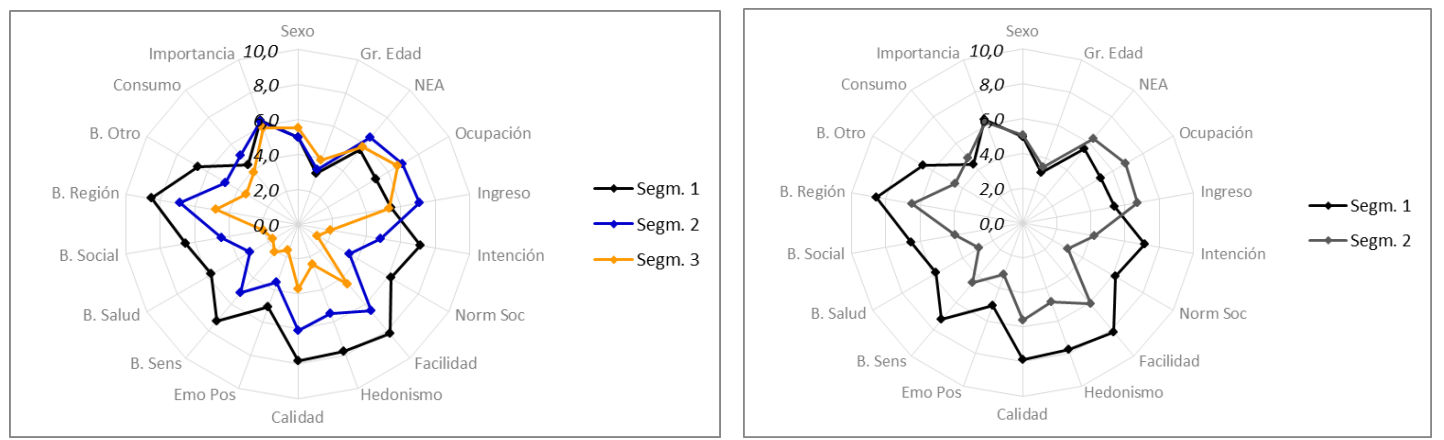

Figuras 4-5. Comportamiento de variables según segmento ( 3 y 2 segmentos respectivamente). Fuente: elaboración propia por parte de los autores. 


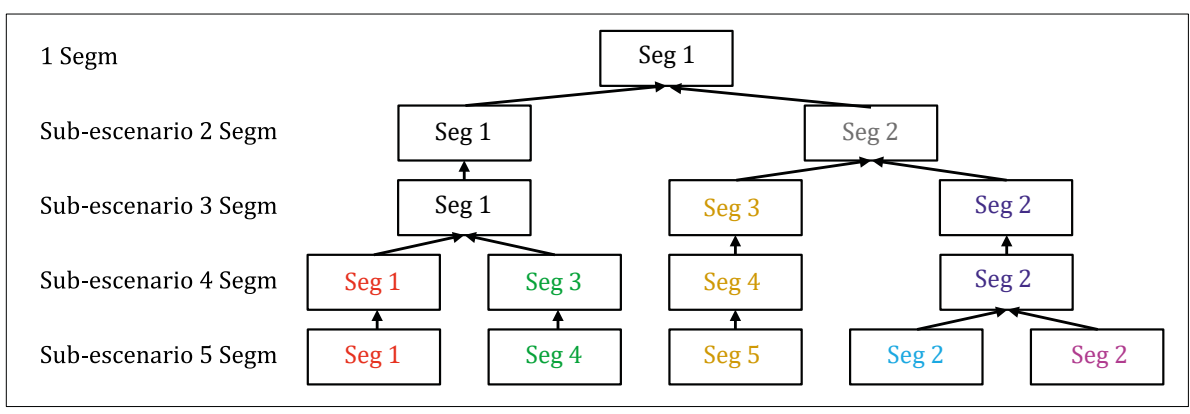

Figura 6. Secuencia de conformación de segmentos. Fuente: elaboración propia por parte de los autores.

Tabla 1. Total de personas por segmento según subescenario (escenario VII)

\begin{tabular}{lcccccc}
\hline \multirow{2}{*}{ Subescenario } & \multicolumn{7}{c}{ Segmento } \\
\cline { 2 - 7 } & Total & 1 & 2 & 3 & 4 & 5 \\
\hline VII.1 & 500 & 500 & - & - & - & - \\
\hline VII.2 & 500 & 257 & 243 & - & - & - \\
\hline VII.3 & 500 & 257 & 192 & 51 & - & - \\
\hline VII.4 & 500 & 138 & 192 & 119 & 51 & - \\
\hline VII.5 & 500 & 138 & 58 & 134 & 119 & 51 \\
\hline
\end{tabular}

Fuente: elaboración propia por parte de los autores.

Para determinar el mejor caso se contrastaron los promedios de cada una de las 19 variables entre segmentos con todas las posibles combinaciones dos a dos, a saber: para 2 segmentos 1 contraste posible (segm. 1 frente segm. 2 ); 3 contrastes para 3 segmentos (segm. 1 frente segm. 2; segm. 1 frente segm. 3; segm. 2 frente segm. 3); con 4 segmentos 6 contrastes y con 5 segmentos 10 contrastes. Se clasificaron las diferencias en 5 categorías (nula, débil, media, fuerte y muy fuerte) en función de la cantidad de diferencias significativas encontradas sobre el total de contrastes, con confianzas de 0,95, y 0,90 . Se encontraron mayores diferencias con 4 segmentos, de modo que este es el escenario recomendado. En la siguiente tabla se muestra la mencionada clasificación.

Tabla 2. Clasificación de diferencias significativas entre variables de diferentes segmentos según subescenario

\begin{tabular}{|c|c|c|c|c|c|c|c|c|c|c|c|c|c|c|c|c|c|c|c|}
\hline \multirow[t]{2}{*}{ Subescenario } & \multicolumn{19}{|c|}{ Variable } \\
\hline & 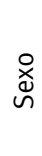 & 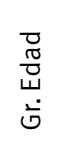 & 岕 & 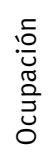 & 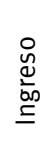 & 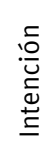 & $\begin{array}{l}\text { O } \\
\text { ஸे } \\
\text { E } \\
\text { O }\end{array}$ & 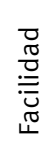 & 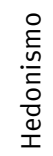 & $\begin{array}{l}\frac{0}{0} \\
\frac{0}{0} \\
\tilde{U}\end{array}$ & 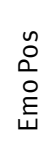 & 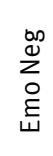 & $\begin{array}{l}\tilde{n} \\
\tilde{D} \\
\sim \\
\infty\end{array}$ & $\begin{array}{l}\frac{0}{3} \\
\stackrel{0}{n} \\
\infty\end{array}$ & $\begin{array}{l}\bar{\pi} \\
\frac{\pi}{U} \\
\text { ज } \\
\infty\end{array}$ & 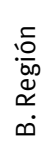 & $\begin{array}{l}\text { 옴 } \\
\text { ஸे } \\
\dot{0}\end{array}$ & 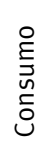 & 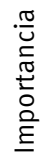 \\
\hline 2 Segm & 0 & D & $\mathrm{F}$ & MF & $\mathrm{F}$ & MF & MF & MF & MF & MF & MF & $\mathrm{F}$ & MF & MF & MF & MF & MF & 0 & 0 \\
\hline 3 Segm & 0 & $M$ & $M$ & $\mathrm{~F}$ & $M$ & MF & MF & MF & MF & MF & MF & $\mathrm{F}$ & $\mathrm{MF}$ & MF & MF & $\mathrm{MF}$ & MF & $\mathrm{D}$ & D \\
\hline 4 Segm & 0 & MF & M & MF & M & MF & MF & MF & MF & MF & MF & MF & MF & MF & MF & MF & MF & $\mathrm{F}$ & $\mathrm{F}$ \\
\hline 5 Segm & D & M & D & $\mathrm{F}$ & M & MF & MF & MF & MF & MF & MF & M & MF & MF & MF & MF & MF & M & M \\
\hline
\end{tabular}

Nota: $\mathrm{O}=$ nula; $\mathrm{D}=$ débil; $\mathrm{M}=$ media; $F=$ fuerte y $M F=$ muy fuerte. 
Por último, se muestran las descripciones de cada segmento:

(i) Optimista: el más abierto a la innovación; el más joven; consumo de vino medio; le confiere importancia a la compra del vino; predominio de desempleados y estudiantes; nivel educativo medio bajo e ingresos bajos. Dispuesto a probary consumir novedades (como, por ejemplo, el vino tinto espumoso en cuestión), aunque por sus bajos ingresos debe ser a un precio económico.

(ii) Dispuesto: abierto a la innovación, pero menos que el optimista; adulto joven; consumo de vino bajo; le confiere moderada importancia a la compra del vino; predominio de amas de casa, estudiantes y jubilados; nivel educativo medio bajo e ingresos bajos. Candidato a consumir el producto, pero igual que el optimista debe ser a un precio económico debido a sus bajos ingresos y consumo.

(iii) Exigente moderado: exigente y poco abierto a la innovación; adulto joven; alto consumo de vino; presta importancia a la compra del vino; predominan funcionarios, empresarios y empleados; con nivel educativo e ingresos altos. Puede comprar un producto de precio alto, pero su propensión a la compra no es buena.

(iv) Pretencioso: el menos abierto a la innovación; adulto, el de mayor edad; bajo consumo de vino, el menor; moderada importancia a la compra del vino; jubilados, profesionales y autónomos; con nivel educativo e ingresos bajos. Igual que el exigente, no es un potencial comprador.

\section{Conclusiones}

Ante la complejidad del mercado del vino, aspectos tales comola innovación y segmentación del mercado cobran primordial importancia para el éxito de los productores; una segmentación apropiada permite identificar grupos de individuos diferentes y enfocar la innovación hacia el o los grupos idóneos para el producto, y así mejorar su introducción.

En este sentido se segmentó el mercado de acuerdo al comportamiento de los individuos ante un nuevo vino tinto espumoso natural en función de variables como son las actitudes, comportamientos en el consumo del vino, beneficios y emociones causadas, edad, género, nivel educativo finalizado e ingresos. Las técnicas estadísticas como el alfa de Cronbach (para verificar la consistencia de los datos), el ACP (para reducción de la cantidad de variables y que esas variables faciliten la conformación de los segmentos) y el AC (para segmentar) permiten afirmar que existen distintos grupos y que no todos los consumidores de vino se comportan de igual manera ante una innovación.

Luego de analizar cada escenario se seleccionó 1 de 4 segmentos, ordenados desde el más propenso a consumir la nueva bebida hasta el que menos (siendo estos el optimista, dispuesto, exigente moderado y pretencioso).

Respondiendo a la pregunta planteada, ¿a quién dirigir el vino novedoso?, la respuesta sin duda es al segmento 'optimista'; ya que este segmento es el más abierto y propenso al consumo del vino con un marketing apropiado con el cual se sienta identificado; mientras que en segundo lugar al segmento 'dispuesto'. Ambos se caracterizan por ser jóvenes o "adulto joven" con consumo de vino de medio a bajo, desempleados o jubilados con bajos ingresos.

La metodología adoptada es apropiada para determinar los posibles nichos de mercado, discriminando individuos de acuerdo a sus características y posición ante nuevos productos; ello resulta en un apoyo a la gestión de bodegas, cooperativas, asociaciones de bodegas 0 denominaciones de origen, de cara al lanzamiento de nuevos productos. El siguiente paso será cuantificar el tamaño de cada segmento.

\section{Referencias}

Barrena, R., García, T. y López-Mosquera, N. (2016). Determinantes socioeconómicos y emocionales en el consumo de nuevos alimentos. Un estudio piloto. ITEA, 112(1), 88-103.

Blashfield, R.K. (1976). Mixture model tests of cluster analysis: Accuracy of four agglomerative hierarchical methods. Psychological Bulletin, 83(3), 377-388. 
Ferreira, L. and Hitchcock, D.B. (2009). A Comparison of Hierarchical Methods for Clustering Functional Data. Communications in Statistics-Simulation and Computation, 38(9), 1925-1949.

Frías-Navarro, D. (2014). Apuntes de SPSS. Valencia, España: Universidad de Valencia.

García-Milon, A. et al. (2019). Does the product test really make a difference? Evidence from the launch of a new wine. Food Quality and Preference, 71, 422-430.

George, D. and Mallery, P. (2003). SPSS for Windows step by step: A simple guide and reference, 11.0 update. Boston, USA: Allyn \& Bacon.

Gliem, J.A. and Gliem, R. (2003). Calculating, Interpreting, and Reporting Cronbach's Alpha Reliability Coefficient for Likert-Type Scales. Recuperado de https://scholarworks.iupui. edu/handle/1805/344.

Gourville, J.T. (2006). Eager Sellers and Stony Buyers: Understanding the Psychology of NewProduct Adoption. Harvard Business Review, 84(6), 98-106.

Granato, D. etal. (2018). Use of principal component analysis (PCA) and hierarchical cluster analysis (HCA) for multivariate association between bioactive compounds and functional properties in foods: A critical perspective. Trends in Food Science \& Technology, 72, 83-90.

Hands, S. and Everitt, B. (1987). A Monte Carlo study of the recovery of cluster structure in binary data by hierarchical clustering techniques. Multivariate Behavioral Research, 22(2), 235-243.

Juaneda-Ayensa, E. et al. (2019). The "right" wine taster: Identifying individuals high in emergent nature to develop new market-oriented products. British Food Journal, 121(3), 675-696.

Khodasevich, M.A. et al. (2017). Identification of Counterfeit Alcoholic Beverages Using Cluster Analysis in Principal-Component Space.Journal of Applied Spectroscopy, 84(3), 517-520.
Kuiper, F.K. and Fisher, L. (1975). A Monte Carlo comparison of six clustering procedures. Biometrics, 31(3), 777-783.

Moreno, M. y Munuera, J.L. (2012). La revisión del conocimiento en los nuevos productos: el papel mediador de la creatividad y la velocidad al mercado. Revista Española de Investigación de Marketing ESIC, 16(1), 59-85.

OEMV. (2014). El consumo de vino en España. Recuperado de http://www.oemv.es/ infografia-oemv-el-consumo-de-vino-enespana.

OEMV.(2018). El número de bodegas en España sube hasta las 4.373. Recuperado de http://www. oemv.es/el-numero-de-bodegas-en-espanasube-hasta-las-4-373.

OIV. (2018). Bases de datos y estadísticas. Recuperado de http://www.oiv.int/es/basesde-datos-y-estadisticas/estadisticas.

OIV. (2018). Aspectos de la coyuntura mundial. Recuperado de http://www.oiv.int/public/ medias/5960/aspectos-de-la-coyunturamundial-oiv-abril-2018.pdf.

Olarte, C., Pelegrín, J. and Reinares, E. (2017). Model of acceptance of a new type of beverage: Application to natural sparkling red wine. Spanish Journal of Agricultural Research, 15(1), e0102.

Smith, W.R. (1956). Product differentiation and market segmentation as alternative marketing strategies. Journal of Marketing, 21(1), 3-8.

Watson, D., Clark, L. and Tellegen, A. (1988). Developmentand Validation of Brief Measures of Positive and Negative Affect: The PANAS Scales. Journal of Personality and Social Psychology, 54(6), 1063-1070. 